\title{
ACTIVIDADES EN LABORATORIOS DE ENSEÑANZA DE FÍSICA: PERCEPCIONES DE ESTUDIANTES DE BRASIL Y ARGENTINA. TEMA DE REFLEXIÓN DOCENTE
}

\author{
Liliana del Valle Ortigoza \\ LILIANA Ester CONTINI \\ Universidad Nacional del Litoral (UNL), Santa Fe, Argentina \\ Marcel da Silva Lessa de Oliveira \\ Universidade Estadual de Santa Cruz (UESC), Ilhéus, Bahia, Brasil
}

\begin{abstract}
RESUMEN: En este trabajo se reflexiona sobre percepciones y representaciones que alumnos de universidades de Brasil y Argentina poseen sobre el trabajo en el laboratorio docente de Física. Participaron 150 alumnos que cursan laboratorios presenciales de Física de UESB y UESC (Brasil) y UNL (Argentina). Cerca del 100\% de los encuestados refiere utilizar guías preestablecidas en el trabajo experimental mientras que un bajo porcentaje (30\% de Brasil y $12 \%$ de Argentina) reconoce el uso de TIC; respecto a sus opiniones sobre el objetivo de las actividades de laboratorio de Física, $87 \%$ y 96\% respectivamente, entiende que se trata de Aplicar/Reforzar/ Verificar la teoría aprendida. En ambos países, la mayoría, refiere no haber utilizado nunca o no lo recuerdan, experiencias en sus clases de Física en niveles educativos precedentes.
\end{abstract}

Palabras clave: Enseñanza de Física. Laboratorio didáctico. Percepciones. 
INTRODUCCIÓN

Dada la importancia atribuida por profesores y alumnos a los trabajos prácticos de ciencias, según lo mencionado por diversas investigaciones (GUILLARÓN et al., 2012; FURIÓ; PAYÁ; VALDÉS, 2005; MILLAR; OSBORNE, 1998), su utilización en la enseñanza de ciencias es ampliamente defendida. Mucho se ha escrito sobre la importancia y forma de utilización de los experimentos.

Distintos autores consideran fundamental que en el proceso educativo se desarrollen actividades prácticas que permitan fortalecer los conocimientos teóricos y estimulen a los estudiantes en la investigación, promoviendo reflexión, crítica y desarrollo de pensamiento científico (BORGES, 2002; LIMÓN; CARRETERO, 1997; TAMAYO; SANMARTÍ, 2007; TAMAYO, 2009). Desde el punto de vista del constructivismo, la actividad experimental desempeña un rol muy importante si se dirige de manera consciente e intencionada a lograr que las ideas previas de los estudiantes evolucionen a conceptos más elaborados y cercanos a los científicos (TAMAYO; SANMARTÍ, 2007; TAMAYO, 2009).

En este sentido, Andrade (2013) sostiene que las investigaciones en la enseñanza de ciencias ya tienen como consenso que el principal objetivo de los trabajos prácticos es promover los siguientes aprendizajes:

De contenidos científicos;

De la naturaleza del conocimiento científico;

Del abordaje de experimentos a partir de resolución de problemas.

Pero, para lograr alcanzar estos objetivos, es necesario que las actividades sean planeadas y ejecutadas adecuadamente. Además de los factores externos a los profesores (equipamientos de laboratorio, espacios para realizar las actividades, tiempo disponible en los cursos de ciencias, entre otros) esto también depende de las concepciones que poseen docentes y alumnos acerca de los objetivos de los trabajos prácticos (BARBERÁ; VALDÉS, 1996).

Andrade, Becker y Vainstein (2013) señalan que los profesores en general tienen concepciones epistemológicas empiristas, según las cuales el conocimiento es producido a partir de la observación, siguiendo un método riguroso de proceder. Esta idea hace que las actividades prácticas se enfoquen en los resultados a alcanzar, en detrimento del proceso. Como consecuencia, no se confiere a los alumnos libertad para elegir como proceder, ya que los resultados y la forma de obtenerlos son conocidos previamente.

Así, según Carrascosa, Gil-Pérez y Vilches (2006), se observa que cuando los profesores utilizan trabajos prácticos, tienen la tendencia de diseñarlos como "recetas de cocina". De esta forma, en lugar de desarrollar una visión 
realista de la naturaleza de la ciencia y adaptar a los estudiantes a manejar los experimentos, en verdad crean o refuerzan concepciones equivocadas que reflejan en sus estudios universitarios (FURIÓ; PAYÁ; VALDÉS, 2005).

Más allá de las discusiones acerca del aprendizaje a partir de esta metodología, se observa claramente una inadecuación en el caso de carreras de grado del área de ciencias y tecnología, ya que estos futuros profesionales se desempeñarán en laboratorios, donde se supone que enfrentarán constantemente situaciones nuevas y abiertas. Otro aspecto problemático es el hecho de que, en situaciones del cotidiano que involucran el uso de conocimientos científicos, tampoco hay respuestas y procedimientos conocidos a priori. Tales características tornan inadecuadas este tipo de actividades utilizadas con elevada frecuencia para trabajar las interacciones entre ciencia, tecnología y sociedad, defendidas ampliamente en la literatura (FERNÁNDEZ et al, 2005).

Por lo anterior y por otras cuestiones como la promoción de espíritu reflexivo, crítico y autogestión del conocimiento, diversos autores sostienen que los trabajos prácticos deben seguir un modelo de investigación orientada, o sea, enfocar la búsqueda de resolución a un problema. En este proceso, los estudiantes no solo deben generar hipótesis acerca del problema, sino también participar activamente en el planeamiento del diseño experimental a ser utilizado. De esta forma, ellos pueden percibir que la actividad científica no sigue un método cerrado que empieza por la observación. Al contrario, la recolección de datos es orientada por las hipótesis y por el cuerpo de conocimientos que dan base a la investigación (CARRASCOSA; GIL-PÉREZ; VILCHES, 2006).

No obstante, dar mayor libertad a los alumnos en un proceso de investigación orientada exige de ellos habilidades que no siempre poseen cuando llegan a la universidad (lo que, a su vez, está relacionado a la utilización o no de actividades experimentales en los niveles educativos precedentes). Las concepciones que los estudiantes poseen sobre la temática en estudio, influencian las expectativas acerca de los trabajos prácticos en la universidad.

Por lo tanto, es necesario investigar cuáles son las concepciones epistemológicas y pedagógicas que están involucradas en la realización de estas actividades, tanto por parte de los docentes como de los estudiantes. Conocer las percepciones y representaciones que los alumnos tienen sobre esta actividad es un paso hacia la comprensión y mejora de la enseñanza y consecuentemente del aprendizaje. A partir de la mirada de los estudiantes se pueden interpretar los procesos de enseñanza, evaluando lo que efectivamente ocurre en el ámbito del laboratorio docente (HERRÁN, 2011). 
Lo anterior se constituye en insumo importante para el diseño de actividades que promuevan el interés por la ciencia, su enseñanza y aprendizaje.

El presente trabajo investiga percepciones de alumnos de distintas carreras de diferentes universidades, sobre las actividades en el laboratorio experimental. Lo realizado forma parte de actividades en el marco de dos programas de posgrado de Brasil y Argentina, que promueve anualmente el intercambio de profesionales entre las instituciones participantes: Universidade Estadual do Sudoeste da Bahia (UESB) y Universidade Estadual de Santa Cruz (UESC), Brasil, y Universidad Nacional del Litoral (UNL), Santa Fe, Argentina.

\section{Objetivos}

Analizar percepciones de alumnos universitarios respecto del trabajo en el laboratorio presencial de Física, en ámbitos universitarios de Brasil y Argentina.

Contribuir a la reflexión acerca de metodologías utilizadas en las clases de laboratorio

\section{Metodología}

ACERCA DE LA MUESTRA DE ESTUdiO:

En el presente estudio participaron cinco carreras de tres universidades, dos de Brasil, una de UESC y otra de UESB y tres de Argentina, de UNL. La investigación se desarrolló en el marco del convenio Programa de Pós-Graduação em Memória: Linguagem e Sociedade, entre dos de las citadas universidades, UESB y UNL, que propicia el intercambio entre investigadores de los dos países, al realizar periódicamente misiones de estudio. Ya que este trabajo busca investigar las percepciones acerca de las clases de laboratorio presencial de Física, fueron elegidas carreras que involucran materias con la mencionada metodología de trabajo.

Destacamos que, en UESC la carrera de Física se divide en profesorado y licenciatura, en cuanto en UESB todos son alumnos de profesorado, en su totalidad carreras de área Física. 
Las carreras participantes de la UNL, pertenecientes al área de Ciencias Biológicas y de la Salud, presentan una duración de cinco años con un ciclo básico donde la disciplina Física tiene marcada incidencia. En Licenciatura en Nutrición la materia Física General y Termodinámica corresponde al segundo semestre del primer año de la carrera, mientras que en Bioquímica y Licenciatura en Biotecnología la materia Física II corresponde al segundo semestre del segundo año de las carreras, siendo esta materia de cursado común para las dos carreras mencionadas.

\section{ACERCA DEL INSTRUMENTO UTILIZADO:}

Para conocer las percepciones de los estudiantes, fue elaborado un cuestionario mixto ad hoc, con 5 preguntas de opción múltiple y 8 preguntas abiertas. Las siete primeras tratan de caracterizar el sujeto (en qué carrera estudia, tiempo de ingreso, experiencias con la enseñanza de Física), saber si ha tenido actividades experimentales en las instituciones donde ha estudiado en niveles educativos precedentes, y conocer sus relaciones con la enseñanza de Física. Las otras seis son todas abiertas y buscan conocer sus opiniones acerca de cómo ha realizado los trabajos prácticos de Física en su carrera, y cuáles son (o deberían ser, en su opinión) los objetivos de los mismos.

El cuestionario fue previamente sometido a una prueba piloto para su validación.

ACERCA DEL PROCESAMIENTO DE DATOS: (PROGRAMA, VARIABLES)

El análisis de los datos obtenidos consistió de tres etapas principales:

- Caracterización de la muestra de investigación, a partir de preguntas de opción múltiple

- Planteo de categorías que abarquen las principales concepciones y representaciones observadas

- Análisis estadístico: los datos de las encuestas se sistematizaron y cargaron en el software estadístico SPSS 21.0, disponible en la Facultad de Bioquímica y Ciencias Biológicas, UNL -Argentina-. Se resumió la información mediante tablas, gráficos y medidas de resumen estadístico adecuadas al tipo de variable analizada. Para la comparación de medias se utilizó la prueba t de Student y para la comparación de proporciones o independencia, la prueba c2 o la prueba Fisher exacta. El nivel de significación estadística adoptado fue 0,05 (ELORZA PEREZ-TEJADA, 2010). 
A partir de los resultados de este análisis, se investiga si existen relaciones entre las percepciones de los alumnos y variables como la carrera que está cursando, el uso (o no) de actividades experimentales en niveles educativos no universitarios, su intención (o no) de enseñar Física en el futuro, entre otras. Lo anterior servirá de sustento a la reflexión acerca de las metodologías utilizadas en laboratorios docentes de Física.

\section{RESULTADOS Y DISCUSIÓN}

Los resultados se analizan en base a las respuestas al cuestionario que se adjunta en Anexo 1.

ACERCA DE LA MUESTRA EN ESTUDIO:

\section{Distribución:}

La muestra de estudio se compone de 150 estudiantes, 30 de la carrera de Física, UESB, 23 de la carrera de Física, UESC, 48 de la carrera de Licenciatura en Nutrición, UNL y 49 de las carreras de Bioquímica y Licenciatura en Biotecnología, UNL, todos ellos participantes voluntarios de la investigación. Aunque estos últimos sean de dos carreras distintas, sus respuestas se estudiaron en un mismo grupo, por que cursan juntos los trabajos prácticos de la asignatura Física. La distribución de la muestra se detalla en Gráfico 1.

Gráfico 1. Distribución de encuestados según carrera y país.

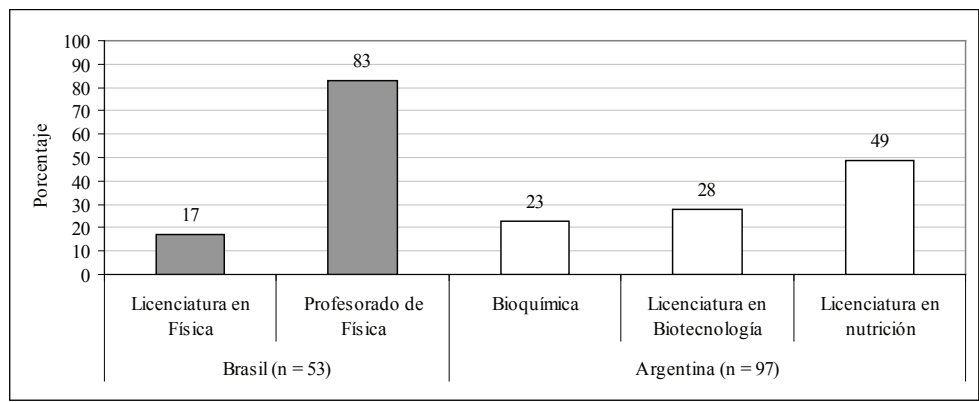

En la gráfica se observa que el número de participantes resultó mayor en Argentina que en Brasil, debido a que las carreras elegidas tienen una 
elevada matrícula. Además esta diferencia puede deberse a que las carreras elegidas de Brasil son de carácter académico y/o investigación, mientras que las de Argentina son carreras con perfil más amplio, ya que incluyen las prácticas profesionales en áreas de Salud e Industria.

\section{Tiempo de Ingreso}

La duración de las carreras mencionadas, en Brasil y Argentina, de acuerdo a los planes de estudios vigentes, es de 5 años.

Con respecto al tiempo de ingreso, la muestra es heterogénea, incluyendo estudiantes de inicio, medio y algunos de final de carrera. El tiempo medio de ingreso es de 1,9 años. En el Gráfico 2 se registra dicha variable según país.

Gráfico 2. Diagrama de cajas Tiempo de Ingreso vs. país.

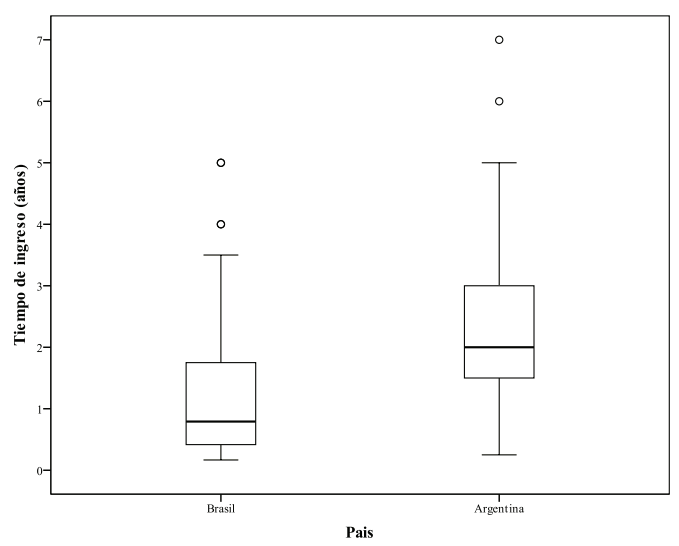

Se destaca del gráfico anterior que los alumnos encuestados de Argentina presentan un tiempo promedio de ingreso a la universidad de 2,21 años que es significativamente mayor que el tiempo promedio de los de Brasil, 1,34 años ( $p<0,001)$.

En Argentina en la última década ha existido un retraso en los tiempos de culminación de carreras de grado universitario, sobre todo en el campo de ciencias exactas y biológicas de universidades estatales, tal como lo comprobaron diversas investigaciones (GARCÍA DE FANELLI, 2014; OSORIO; BOLANCÉ; CASTILLO CAICEDO, 2011; PORTO; DI GRESIA, 2004; VAIRA et. al, 2010), hecho que se corrobora en la presente investigación. 
ACERCA DE EXISTENCIA Y UTILIZACIÓN DE LABORATORIOS EN NIVELES EDUCATIVOS PRECEDENTES:

\section{Existencia de laboratorios en la Enseñanza Secundaria:}

Se indagó acerca de si las instituciones donde los participantes estudiaron en niveles precedentes poseían o no laboratorios de Física. Se observó que en ambos países menos de la mitad de los encuestados manifestó que su escuela secundaria poseía laboratorios de ciencias. En particular se encontró que el 34\% de los 53 encuestados de Brasil respondió afirmativamente y el $41 \%$ de los 97 argentinos. Si bien el porcentaje de colegios con laboratorios de Física de Argentina es mayor que en Brasil, no hay diferencias estadísticamente significativas entre ellos $(p=0,253)$.

\section{Utilización y frecuencia:}

No se encontró asociación estadísticamente significativa entre el país y la frecuencia de uso de experimentos en las clases de Física $(p=0,503)$. Con un alto porcentaje, en ambos países, los encuestados refieren no haber utilizado nunca o no lo recuerdan, experiencias en sus clases de Física. Los resultados se observan en el Gráfico 3.

Gráfico 3. Frecuencia de uso de laboratorio de Física nivel secundario vs. país.

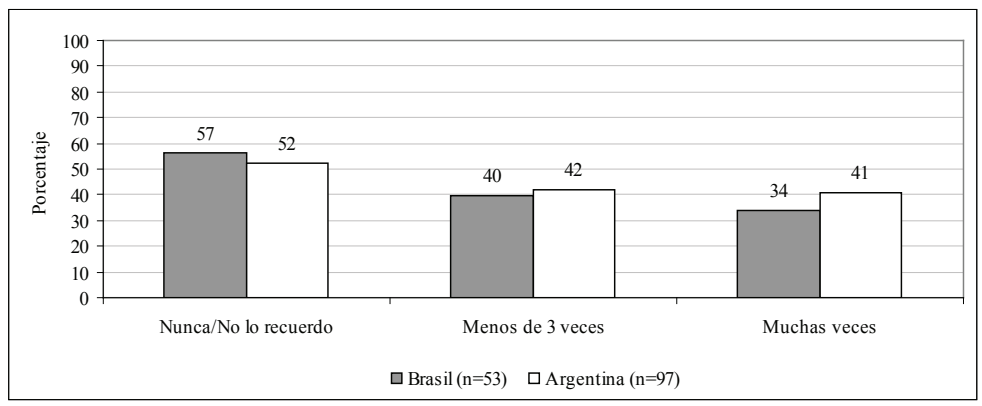

Lo anterior da cuenta de entender la enseñanza de una ciencia experimental de manera netamente teórica, hecho que contradice el discurso acerca de la importancia del trabajo experimental (LEACH, 2002; REIGOSA CASTRO, 2006). 
En un porcentaje próximo al 100\% de los encuestados respondió que sí utilizan guías estructuradas en el trabajo experimental.

Las prácticas de laboratorio constituyen un elemento importante del proceso integral de construcción del conocimiento científico, demandando un esfuerzo creativo y crítico por parte de los estudiantes, evitando reducirse a directivas que impongan caminos preestablecidos, inmodificables o incuestionables (GARCÍA; TUÑON, 2005; SALINAS; GIL; CUDMANI, 1995). En esta dirección, tal como menciona Carrascosa et al. (2006), se supone el aprendizaje de las ciencias como significativo en la medida en que el estudiante se identifica con las actividades desarrolladas durante el proceso de enseñanza, considerando apropiado la utilización de trabajos de laboratorio que superen la simple puesta en práctica de guías preestablecidas.

¿Puede lograrse lo anterior si la totalidad de los encuestados refiere al uso de guías preestablecidas estructuradas?

Al solicitar a los estudiantes que sigan una guía detallada, se contribuye a una visión rígida, algorítmica y cerrada de la ciencia. Conviene insistir en que resulta de fundamental importancia que los estudiantes tengan ocasión de participar en la elaboración de diseños experimentales en vez de seguir guías estructuradas, considerando esta etapa relevante en todo proceso de investigación y de utilidad para comprender las relaciones entre ciencia y tecnología.

\section{ACERCA DE UTILIZACIÓN DE INNOVACIONES TECNOLÓGICAS}

De los 150 encuestados contestaron esta pregunta solo 127 (83 argentinos, 44 brasileros). Se encontró que solo el 12\% de los estudiantes argentinos consideran que si hay innovaciones tecnológicas en sus prácticas de laboratorio, en contraste con el $30 \%$ de Brasil, pudiéndose considerar que el porcentaje de Brasil es significativamente mayor que el de Argentina $(p=0,015)$.

A pesar de que el porcentaje resulta significativamente mayor en Brasil que en Argentina, es notorio el bajo número de estudiantes que consideran que en sus prácticas de laboratorio, se incluyen innovaciones tecnológicas.

¿Indica lo anterior que se sigue manteniendo una práctica tradicional de enseñanza de Física en el laboratorio, sin utilizar las tecnologías de la información y la comunicación?

¿Se utilizarán las TIC pero los estudiantes no alcanzan a percibirlo, debido a su condición de nativo digital? 
Gráfico 4. Utilización de innovaciones tecnológicas vs. país.

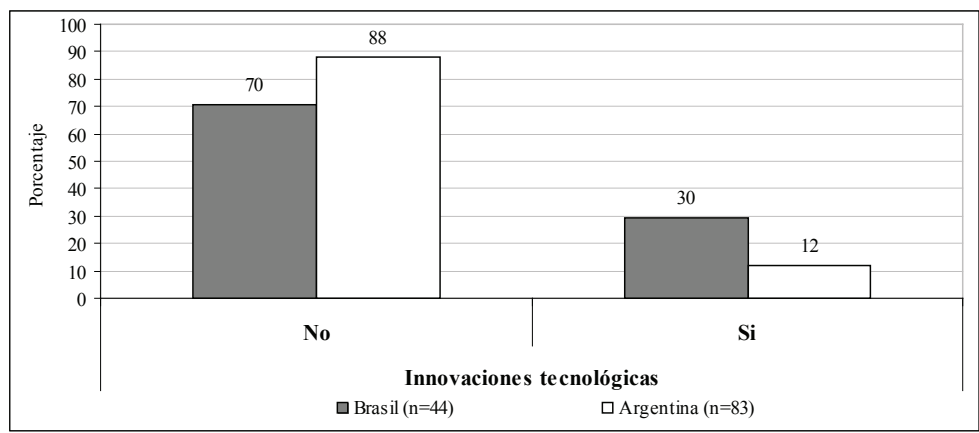

Gras Martí y Cano Villalba (2003) mencionan que el manejo de TIC se realiza en el trabajo de laboratorio desde el principio, a base del tratamiento y procesamiento de imágenes, hojas de cálculo, programas de adquisición de datos, entre otros. Lo mencionado no es percibido por los alumnos como utilización de tecnologías, probablemente se origine en la condición de nativo digital propia de los estudiantes del siglo XXI, donde la concepción de TIC se enfocaría al uso de redes y programas sofisticados.

\section{ACERCA DEL OBJETIVO DE LAS DISCIPLINAS CON LABORATORIOS PRESENCIALES}

En base a las respuestas a la mencionada pregunta se proponen y precisan las siguientes categorías:

Aplicar/Reforzar/Verificar teoría aprendida

Aprender contenidos

Aproximación a lo cotidiano

En el Gráfico 5 se detallan los porcentajes de respuestas de acuerdo a la categoría y país del estudiante.

Se destaca el elevado porcentaje de alumnos (87\% Brasil; $96 \%$ Argentina) que manifiesta como objetivo de las clases de laboratorio de Física Aplicar/Reforzar/Verificar la teoría aprendida, pudiéndose considerar que el porcentaje de alumnos de Argentina que considera esto es significativamente mayor que el de Brasil ( $p=0,046)$; en contraposición un muy bajo porcentaje de alumnos (12\% Brasil; 22\% Argentina) sostiene como objetivo de las clases de laboratorio aproximar la teoría a la vida cotidiana; en este caso si bien el porcentaje de alumnos argentinos es numéricamente mayor, la diferencia observada no alcanza a ser significativa estadísticamente $(p=0,165)$. 
Gráfico 5. Objetivos de trabajos de laboratorio vs. país.

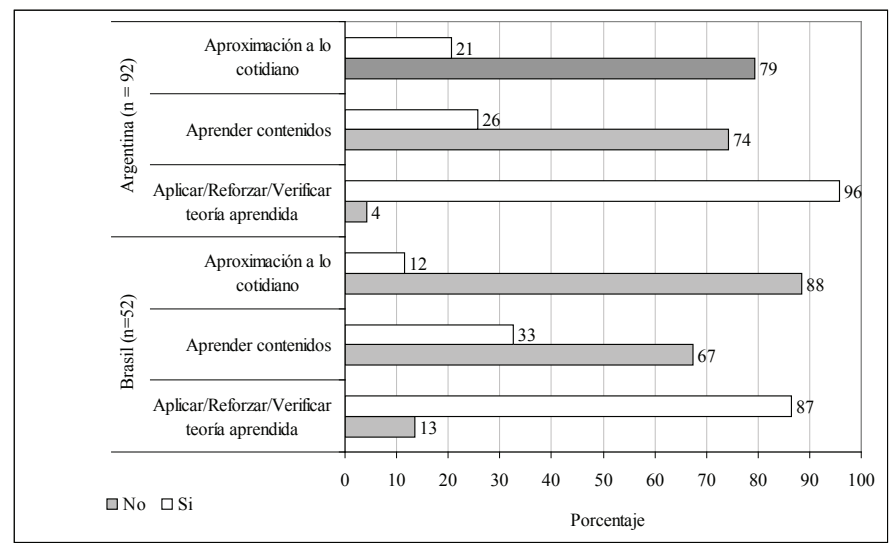

Solbes y Vilches (2006) Ilaman la atención sobre la importancia de establecer relaciones entre la ciencia y los procesos de la vida cotidiana. ¿De qué manera se puede propiciar encontrar dichas relaciones? ¿En qué contexto se logrará otorgar un nuevo sentido a la práctica, a fin de motivar a los estudiantes a ver la ciencia de una manera más amplia donde se emplean materiales cotidianos que les permitan promover aprendizajes significativos?

RELACIONES ENTRE PERCEPCIONES DE LOS ESTUDIANTES Y VARIABLES

USO DE LABORATORIO EN NIVELES EDUCATIVOS PRECEDENTES Y OBJETIVOS DEL LABORATORIO PRESENCIAL:

Se investigó la asociación entre el haber usado o no laboratorios en niveles educativos anteriores y la percepción sobre el objetivo de las disciplinas con laboratorios presenciales. Los resultados se presentan en la tabla 1. Para el análisis de los resultados las categorías de utilización y frecuencia de uso del laboratorio de Física en niveles educativos precedentes se agruparon en 2: -al menos una vez y -Nunca/no recuerdo.

De los resultados obtenidos se observa independencia entre uso de laboratorio en niveles precedentes y percepciones sobre los objetivos del mismo ( $p \geq$ que 0,1605 ). 
Tabla 1. Asociación entre objetivos mencionados y usos de laboratorio.

\begin{tabular}{|l|l|l|l|l|l|l|}
\hline \multirow{2}{*}{$\begin{array}{l}\text { Usaste } \\
\text { laboratorio } \\
\text { en nivel } \\
\text { medio }\end{array}$} & \multicolumn{2}{|l|}{$\begin{array}{l}\text { Aprender contenidos } \\
(\mathbf{n = 1 4 5 )}\end{array}$} & \multicolumn{2}{|l|}{$\begin{array}{l}\text { Aplicar/reforzar/ verificar } \\
\text { teoría aprendida (n=144) }\end{array}$} & \multicolumn{2}{l|}{$\begin{array}{l}\text { Aproximación a lo } \\
\text { cotidiano (n=145) }\end{array}$} \\
\cline { 2 - 7 } & No & Si & No & Si & No & Si \\
\hline $\begin{array}{l}\text { Al menos vez } \\
\text { una }\end{array}$ & 48 & 21 & 3 & 66 & 59 & 10 \\
\hline $\begin{array}{l}\text { Nunca/no } \\
\text { recuerdo }\end{array}$ & 56 & 20 & 8 & 68 & 60 & 15 \\
\hline Valor $\mathbf{p}$ & 0,582 & & 0,161 & & 0,383 & \\
\hline
\end{tabular}

DESEMPEÑO FUTURO Y PERCEPCIÓN DE OBJETIVOS DEL LABORATORIO PRESENCIAL:

De los resultados que se observan (tabla II), si bien el $22 \%$ de los estudiantes que ya tienen decidido dedicarse a la enseñanza considera como objetivo del laboratorio la aproximación a lo cotidiano, este porcentaje resulta mayor que el $19 \%$ de los alumnos que no tienen o no decidieron aún enseñar; esta diferencia no resulta estadísticamente significativa $(p=0,495)$.

Tabla II. Asociación entre desempeño futuro y percepción sobre objetivos del laboratorio.

\begin{tabular}{|l|l|l|l|l|l|l|}
\hline $\begin{array}{l}\text { Desempeño } \\
\text { futuro: } \\
\text { Intención } \\
\text { de enseñar } \\
\text { Física }\end{array}$ & \multicolumn{2}{|l|}{$\begin{array}{l}\text { Aprender Contenidos } \\
(\mathbf{n}=145)\end{array}$} & \multicolumn{2}{l|}{$\begin{array}{l}\text { Aplicar/reforzar/ Verificar } \\
\text { teoría aprendida (n=144) }\end{array}$} & $\begin{array}{l}\text { Aproximación a lo } \\
\text { Cotidiano (n=145) }\end{array}$ \\
\cline { 2 - 7 } & No & Si & No & Si & No & Si \\
\hline SI & 18 & 14 & 8 & 24 & 25 & 7 \\
\hline $\begin{array}{l}\text { NO/no lo } \\
\text { decidí }\end{array}$ & 86 & 27 & 3 & 110 & 94 & 18 \\
\hline Valor $p$ & 0,027 & & 0,001 & & 0,495 & \\
\hline
\end{tabular}

El 75\% de los estudiantes que han decidido enseñar piensa que el laboratorio tiene como objetivo Aprender/Reforzar/Verificar teoría aprendida, mientras que el $97 \%$ de los restantes presenta la misma percepción, siendo este último porcentaje significativamente mayor $(p=0,001)$.

El elevado porcentaje de estudiantes -futuros educadores- que considera que las prácticas de laboratorio solo sirven para comprobar la teoría sin dar lugar a otro tipo de experiencias prácticas es un hecho preocupante ya que coincide con una de las mayores críticas realizadas a los trabajos prácticos en la enseñanza tradicional, que conlleva a una visión cientificista de la ciencia. 
Coincidiendo con Osorio (2004), en esta investigación se entiende que la actividad experimental no debe ser vista sólo como una herramienta de conocimiento. También debe considerarse como un instrumento que promueve objetivos conceptuales, procedimentales y actitudinales que debe incluir cualquier dispositivo pedagógico.

\section{Conclusiones}

ACERCA DE LAS CATEGoRIÁS encontradAS:

Un elevado porcentaje de encuestados de ambos países, refiere no haber desarrollado en la educación secundaria clases en laboratorios presenciales de Física, lo que evidencia la falta de infraestructura y/o el enfoque teórico de la enseñanza disciplinar en niveles educativos precedentes.

Con respecto a objetivos de las clases de laboratorios presenciales un elevado porcentaje de alumnos de Argentina entiende que se trata de Aplicar/ Reforzar/Verificar la teoría aprendida, resultando significativamente mayor que para los estudiantes de Brasil. En contraposición un muy bajo porcentaje de alumnos, similares en ambos países, sostiene como objetivo de las clases de laboratorio aproximar la teoría a la vida cotidiana.

Respecto a la utilización de guías preestablecidas, los estudiantes de Brasil y Argentina manifiestan utilizar guías preestablecidas y acuerdan con esta metodología. Coinciden también en considerar escasa la utilización de TIC en el laboratorio didáctico.

\section{ACERCA DE LAS RELACIONES ENTRE PERCEPCIONES Y CATEGORÍAS}

Se observa independencia entre uso de laboratorio en niveles educativos precedentes y percepciones sobre los objetivos del mismo.

Un elevado porcentaje de estudiantes que han decidido enseñar (75\%) menciona que el laboratorio tiene como objetivo Aprender/Reforzar/ Verificar teoría aprendida, demostrando una versión cientificista de la ciencia, mientras que solo un $22 \%$ considera como objetivo del laboratorio la aproximación a lo cotidiano.

Adhiriendo con Gil (2014), creemos que las experiencias en laboratorios didácticos de Física tienen como misión fundamental que 
los estudiantes aprendan el camino por el cual se genera el conocimiento científico mismo.

Es así que en el laboratorio se tiene la posibilidad de aprender mediante las experiencias propias, cometiendo errores y reconociéndolos, de manera de estimular la creatividad, la reflexión y la autocrítica.

Encontrar los diversos modos de motivar al alumno a través de actividades experimentales es un desafío concreto de la docencia universitaria, con énfasis en las ciencias experimentales. Conociendo las percepciones de los estudiantes sobre esta temática, se pueden encontrar puntos de articulación entre el hacer en sí y el saber hacer, entendiendo que en la medida en que nos acerquemos al sentir del alumno, se posibilitan nuevas aperturas al proceso de enseñar y de aprender de manera significativa. Si lo anterior se consigue, se podrá promover análisis, reflexión y articulación de conocimientos previos al estudio del fenómeno, con la construcción de nuevo conocimiento.

\section{ATIVIDADES NOS LABORATÓRIOS DE ENSINO DE FÍSICA: A PERCEPÇÃO DE ESTUDANTES DO BRASIL E DA ARGENTINA. UM PENSAMENTO PARA A EDUCAÇÃO}

Resumo: Neste artigo são feitas reflexões sobre as percepções e representações que estudantes universitários no Brasil e na Argentina têm sobre o trabalho no laboratório didático de física. Participaram da pesquisa 150 alunos que cursam disciplinas presenciais de laboratório de física da Universidade Estadual do Sudoeste da Bahia (UESB), Universidade Estadual de Santa Cruz (UESC) (Brasil) e Universidad Nacional del Litoral (UNL) (Argentina). Cerca de 100\% dos entrevistados afirmaram usar roteiros preestabelecidos nas aulas experimentais, enquanto uma porcentagem baixa (30\% no Brasil e $12 \%$ na Argentina) reconhece o uso das Tecnologias da Informação e Comunicação (TIC); em relação aos seus pontos de vista sobre o propósito das atividades de laboratório de física, $87 \%$ e 96\%, respectivamente, considera que se trata de Aplicar/Reforçar/Verificar a teoria aprendida. Em ambos os países, a maioria dos estudantes afirma nunca ter usado ou não se lembrar de ter usado experimentos em suas aulas de física em níveis educacionais anteriores.

Palavras-chave: Ensino de Física. Laboratório didático. Percepções. 
TEACHING ACTIVITIES IN PHYSICS LABORATORIES: PERCEPTIONS OF STUDENTS FROM BRAZIL AND ARGENTINA. A REFLECTION FOR EDUCATION

AвSTRACT: In this paper we reflect on perceptions and representations that students from universities in Brazil and Argentina have about studying in Physics didactic laboratories. 150 students that attend the Physics laboratory classes of Universidade Edtadual do Sudoeste da Bahia (UESB), Universidade Estadual de Santa Cruz (UESC) (Brazil) and Universidad Nacional del Litoral (UNL) (Argentina) participated in the research. Approximately $100 \%$ have reported using pre-established guides in experimental classes while a low percentage (30\% in Brazil and 12\% in Argentina) recognizes the use of Information and Communication Technologies (ICT); regarding their views about the purpose of activities in Physics laboratories, $87 \%$ of students in Brazil and 96\% in Argentina understand that it is about Applying/Reinforcing/Verifying the theory they learned. In both countries, the majority of students expressed that they have never used or do not remember having used experiments in their physics classes in previous educational levels.

KEYWORDs: Physics teaching. Didactic laboratory. Perceptions.

\section{REFERENCIAS}

ANDRADE, J. A. P.; BECKER, M. L. R.; VAINSTEIN, M. H. Atividades experimentais em Ciências à luz da epistemología genética com convergencias em psicología cognitiva e neuropsicologia. In: CHAPANI, D. T.; SILVA, J. S (Org.). Debates em educação científica. São Paulo: Escrituras Editora, 2013. p. 65-77.

BARBERÁ, O.; VALDÉS, P. El trabajo práctico en la enseñanza de las ciencias: una revisión. Enseñanza de las ciencias, v. 14, n. 3, p. 365-379, 1996.

BORGES TARCISO, A. Novos rumos para o laboratório escolar de ciências. Caderno Brasileiro de Ensino de Física, v. 19, n. 3, p. 291-313, 2002.

CARRASCOSA, J.; GIL, D.; VILCHES, A. Papel de la actividad experimental en la educación científica. Cadena Brasilera Enseñanza de la Física, v. 23, n. 2, p. 157-181, 2006.

ELORZA PEREZ-TEJADA, H. Estadística para las Ciencias Sociales: del Comportamiento y de la Salud. 3. ed. Mexico: Cengage Learning Editores, 2008.

FERNÁNDEZ, I. et al. La superación de las visiones deformadas de la ciencia y la tecnología: un requisito esencial para la renovación de la educación científica. In: GIL PÉREZ, D. et al. Como promover el interés por la cultura científica: una propuesta didáctica fundamentada para la educación científica de jóvenes de 15 a 18 años. Santiago: Oficina Regional de Educación de la UNESCO para América Latina y el Caribe - OREALC/UNESCO, 2005. p. 29-62. 
FURIÓ, C.; PAYÁ, J.; VALDÉS, P. ¿Cuál es el papel del trabajo experimental en la educación científica? In: GIL PÉREZ, D. et al. Como promover el interés por la cultura científica: una propuesta didáctica fundamentada para la educación científica de jóvenes de 15 a 18 años. Santiago, Chile: Oficina Regional de Educación de la UNESCO para América Latina y el Caribe - OREALC/UNESCO, 2005. p. 81-102.

GARCÍA DE FANELLI, A. Rendimiento académico y abandono universitario: Modelos, resultados y alcances de la producción académica en la Argentina. Revista Argentina de Educación Superior, RAES, ano 6, n. 8, jun. 2014.

GARCÍA, J. A. G.; TUÑON, M. J. I. Un modelo para la enseñanza de las ciencias. Revista Electrónica de Enseñanza de las Ciencias, v. 4, n. 3, p. 1-20, 2005.

GIL, S. Experimentos de Física: usando las TIC y elementos de bajo costo. Ciudad Autónoma de Buenos Aires: Alfaomega Grupo Editor Argentino, 2014.

GRAS MARTÍ, A.; CANO VILLALBA, M. TIC en La enseñanza de las Ciencias Experimentales. Comunicación y pedagogía: nuevas tecnologías y recursos didácticos, n. 190, p. 39-44, 2003.

GUILLARÓN, J. J. et al. Alcances y limitaciones actuales de la actividad experimental en escuelas de Enseñanza Media de la provincia Santiago de Cuba: criterios de alumnos y profesores. Lat. Am. J. Phys. Educ. v. 7, n. 1, p. 107-117, 2012.

HERRÁN, A. de la. Técnicas didácticas para una enseñanza más formativa. In: ÁLVAREZ AGUILAR, N.; CARDOSO PÉREZ, R. (Org.). Estrategias y metodologías para la formación del estudiante en la actualidad. Camagüey: Universidad de Camagüey, 2011. Disponível em: <https://www.uam.es/personal_pdi/fprofesorado/agustind/ textos/teuniv.pdf>. Acesso em: 16 maio 2016.

HODSON, D. Hacia un enfoque crítico del trabajo de laboratorio. Enseñanza de las Ciencias, v. 12, n. 3, p. 299-313, 1994.

LEACH, J. The use of secondary data in teaching about data analysis in a first year undergraduate biochemistry course. In: PSILLOS, D.; NIEDDERER, H. (Ed.). Teaching and Learning in the Science Laboratory. New York: Kluwer Academic Publishers, 2002. p. 165-178.

LIMÓN, M; CARRETERO, M. Las ideas previas de los alumnos. ¿Qué aporta este enfoque a la Enseñanza de las Ciencias? In: CARRETERO, M. (Org.). Construir y enseñar las Ciencias Experimentales. Buenos Aires: Aique Grupo Editor S.A.,1997. p. 3-18.

MILLAR, R; OSBORNE, J. F. Beyond 2000: science education for the future. London: King's College, 1998.

OSORIO, Y. W. El experimento como indicador de aprendizaje. Boletín PPDQ, n. 43, p. 7-10. 2004.

OSORIO, A.; BOLANCÉ, C.; CASTILLO CAICEDO, M. Deserción y graduación estudiantil universitaria: una aplicación de los modelos de supervivencia. Revista lberoamericana 
de Educación Superior (ries), México, v. III, n. 6, p. 31-57, 2011. Disponível em: <http:// ries.universia.net/index.php/ries/article/view/97>. Acesso em: 4 jun. 2016.

PORTO, A.; DI GRESIA, L. Rendimiento de estudiantes universitarios y sus determinantes. Revista de Economía y Estadística, Cuarta Época, v. 42, n. 1, p. 93-113, 2004.

REIGOSA CASTRO, C. E. Una experiencia de investigación acción acerca de la redacción de informes de laboratorio por alumnos de Física y Química de primero de Bachillerato. Enseñanza de las Ciencias, v. 24, n. 3, p. 325-336, 2006.

SALINAS, J.; GIL, D.; CUDMANI, L. La elaboración de estrategias educativas acorde a un modelo científico de tratar las cuestiones. In: NOVENA REUNIÓN NACIONAL DE EDUCACIÓN EN LA FÍSICA, Salta, APFA, 1995.

SOLBES, J.; VILCHES, A. Papel de las relaciones entre ciencia, tecnología, sociedad y ambiente en la formación ciudadana. Enseñanza de las Ciencias, v. 22, n. 3, p. 337$348,2004$.

TAMAYO, A.; SANMARTÍ, N. "High-School Students' Conceptual Evolution of the Respiration Concept from the Perspective of Giere's Cognitive Science Model". International Journal of Science Education, v. 29, n. 2, p. 215-248, 2007.

TAMAYO A. Didáctica de las ciencias: Evolución conceptual en la enseñanza y el aprendizaje de las ciencias. Manizales: Editorial Universidad de Caldas, 2009.

VAIRA, S. M.; AVILA, O.; WALZ, M. F.; CONTINI, L. E.; RICARDI, P. A. Inserción a la vida universitaria: relación con la Matemática. Aula Universitaria, n. 12, p. 67-78. 2010.

Liliana del Valle Ortigoza: Bioquímica, Magister en Docencia Universitaria, Universidad Nacional del Litoral, Argentina. Docente del Departamento de Física, Facultad de Bioquímica y Ciencias Biológicas, Universidad Nacional del Litoral, Santa Fe, Argentina.

E-mail: ortigoza@fbcb.unl.edu.ar

LILIANA ESTER CONTINI: Ingeniera, Magister en Biometría y Mejoramiento, Universidad de Buenos Aires, Argentina. Docente del Departamento de Matemática, Facultad de Bioquímica y Ciencias Biológicas, Universidad Nacional del Litoral, Santa Fe, Argentina.

E-mail: Icontini@fbcb.unl.edu.ar 
Marcel da Silva LesSa de Oliveira: Mestre em Educação Científica e Formação de Professores pela Universidade Estadual do Sudoeste da Bahia (UESB). Tem experiência na área de Educação, com ênfase em Ensino de Física, atuando principalmente nos seguintes temas: Ensino por Investigação. Professor de Física da Universidade Estadual de Santa Cruz.

E-mail: marcel_s_lessa@hotmail.com 


\section{Anexo 1}
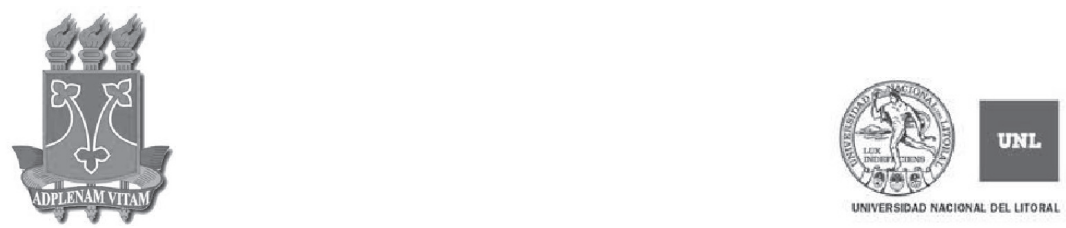

Estimado (a) Alumno (a):

Este cuestionario es parte del proyecto: "Replanteando la Enseñanza de Ciencias y Matemática: Diálogo de las Maestrías en Didáctica de las Ciencias Experimentales (UNL/Argentina) y la Educación la Ciencia y Formación de Profesores (UESB/Brasil)". Este cuestionario trata de reunir información sobre las clases de laboratorio de física en su universidad. Toda la información será de uso exclusivo de los investigadores, para asegurar la confidencialidad. Sus respuestas serán de gran valor para la investigación en curso, por lo que le agradecemos su participación en este proceso.

Marcel da Silva Lessa de Oliveira

Liliana del Valle Ortigoza

1) ¿Cuál es tu carrera?

2) ¿Hace cuánto tiempo ingresaste en la carrera?

3) ¿Has sido profesor de Física? Si [ ] No [ ]

En caso afirmativo, ¿por cuánto tiempo?

4) ¿Has estudiado en algún colegio que tenía laboratorio de ciencias/Física? Si [ ] No [ ]

5) ¿Estudiaste Física con el uso de experimentos durante la educación básica (primaria o secundaria)?

Nunca / No recuerdo [ ] Menos de 3 veces [ ] Algunas veces [ ] Muchas veces [ ]

6) ¿Tienes la intención de enseñar Física en la educación básica después de tu carrera de grado?

Si [ ] No [ ] No lo decidido aún [ ]

7) ¿Si contestaste sí a la pregunta anterior, deseas utilizar experimentos en tus clases? Justifica tu respuesta.

Si [ ]

No [ ]

8) ¿Experimentas con las clases de laboratorio de Física, en la forma en que se desarrollan en tu carrera? ¿Por qué? 
9) ¿Cuál es en tu opinión, lo que es (o lo que debería ser) el principal objetivo de las disciplinas de laboratorio en tu carrera?

10) ¿Con qué frecuencia los profesores de laboratorio utilizan guías de trabajos prácticos (T.P.) en la clase? ¿Consideras que esta herramienta es importante?

11) ¿A lo largo de tu carrera, has identificado algún(a) profesor(a) que realizó/realiza clases experimentales de forma diferente (por ejemplo, usando nuevas metodologías, materiales inusuales, etc)? Si es así, citar ejemplos.

12) En tu opinión, ¿cuál es la influencia de las clases de laboratorio en el aprendizaje del contenido de Física? Justificar.

13) De tu experiencia vivida a lo largo de tu carrera, ¿cuál etapa crees que es más importante en la clase de laboratorio? Justificar.

¡Gracias por tu cooperación! 